\title{
High-energy quark-quark scattering and the eikonal approximation
}

\author{
E. Meggiolaro ${ }^{a}$ \\ ${ }^{\text {a}}$ Dipartimento di Fisica dell'Università and I.N.F.N., Sezione di Pisa, \\ I-56100 Pisa, Italy.
}

The high-energy quark-quark scattering amplitude is calculated first in the case of scalar QCD, using Fradkin's approach to derive the scalar quark propagator in an external gluon field and computing it in the eikonal approximation. The results are then extended to the case of "real" (i.e., fermion) QCD. The high-energy quark-quark scattering amplitude turns out to be described by the expectation value of two lightlike Wilson lines, running along the classical trajectories of the two colliding particles. Interesting analytic properties of the high-energy quark-quark scattering amplitude can be derived, going from Minkowskian to Euclidean theory: they could open the possibility of evaluating the high-energy scattering amplitude directly on the lattice.

It is known that for a class of soft high-energy scattering processes, i.e., elastic scattering processes at high squared energies $s$ in the center of mass and small squared transferred momentum $t$ (that is $s \rightarrow \infty$ and $|t| \ll s$, let us say $\left.|t| \leq 1 \mathrm{GeV}^{2}\right)$, QCD perturbation theory cannot be safely applied, since $t$ is too small. Elaborate procedures for summing perturbative contributions have been developed [1] 2, even if the results are not able to explain the most relevant phenomena.

A non-perturbative analysis, based on QCD, of these high-energy scattering processes was performed by Nachtmann in [3]. He studied the $s$ dependence of the quark-quark (and quarkantiquark) scattering amplitude by analytical means, using a functional integral approach and an eikonal approximation to the solution of the Dirac equation in the presence of an external nonAbelian gauge field.

In a previous paper [4] we proposed an approach to high-energy quark-quark (and quarkantiquark) scattering, based on a first-quantized path-integral description of quantum field theory developed by Fradkin in the early 1960's 5$]$. In this approach one obtains convenient expressions for the full and truncated-connected scalar propagators in an external (gravitational, electromagnetic, etc.) field and the eikonal approximation can be easily recovered in the relevant limit. Knowing the truncated-connected propa- gators, one can then extract, in the manner of Lehmann, Symanzik, and Zimmermann (LSZ), the scattering matrix elements in the framework of a functional integral approach. This method was originally adopted in [6] in order to study the four-dimensional Planckian-energy gravitational scattering.

One starts, for simplicity, with the case of scalar QCD, i.e., the case of a spin-0 quark (described by the scalar field $\phi$ ) coupled to a non-Abelian gauge field $A^{\mu} \equiv A_{a}^{\mu} T_{a}, T_{a}(a=$ $\left.1, \ldots, N_{c}^{2}-1\right)$ being the generators of the Lie algebra of the colour group $S U\left(N_{c}\right)$.

In the Fradkin's approach [5] one writes the propagator for the scalar field $\phi$ as a functional integral of the first quantized theory [7].

The Green function (Feynman propagator) for the scalar field $\phi$ in the external metric $g_{\mu \nu}$ and in the external non-Abelian gauge field $A^{\mu}$ admits a representation in terms of a functional integral over trajectories [5] [6]:

$$
\begin{gathered}
G(y, x \mid g, A)=\int_{0}^{\infty} d \nu \int\left[\sqrt{-g(X)} d X^{\mu}\right] \\
\times \delta(x-X(0)) \delta(y-X(\nu)) \times \\
P \exp \left[i \int_{0}^{\nu} d \tau L(X, \dot{X})\right]
\end{gathered}
$$

where

$$
\begin{aligned}
& L(X, \dot{X})= \\
& \quad \frac{1}{2}\left(g_{\mu \nu} \dot{X}^{\mu} \dot{X}^{\nu} \cdot \mathbf{1}+2 g A_{\mu} \dot{X}^{\mu}-m^{2} \cdot \mathbf{1}\right) .
\end{aligned}
$$


The matrix 1 is the $N_{c} \times N_{c}$ unity matrix in the colour space and " $P$ " means "path-ordered" along the "histories" $X^{\mu}(\tau)$. Moreover $g(X) \equiv$ $\operatorname{det}\left(g_{\mu \nu}(X)\right)$, so that $g(X)=-1$ in a flat spacetime. The propagator (1) is already connected, i.e., vacuum diagrams have been already divided out. Yet, in order to derive the scattering matrix elements following the LSZ approach, we need to know the on-shell truncated-connected Green functions, which are obtained from the connected Green functions by removing the external legs calculated on-shell. In Ref. [6] it is shown how to compute the truncated-connected propagator $\tilde{G}_{t}\left(p, p^{\prime} \mid A\right)$, starting from Eq. (1), in the socalled eikonal approximation, which is valid in the case of scattering particles with very high energy $\left(E \equiv p^{0} \simeq|\mathbf{p}| \gg m\right)$ and small transferred momentum $q \equiv p^{\prime}-p$ (i.e., $|t| \ll E$, where $t=q^{2}$ ). In this limit the functional integral can be evaluated by means of a saddle-point approximation in which the classical trajectory is computed to the lowest non-trivial order, that is a straight line (in the Minkowski space-time) described by a classical particle (of mass $m$ ) moving with fourmomentum $p^{\mu}$ :

$X_{0}^{\mu}(\nu)=b^{\mu}+\frac{p^{\mu}}{m} \nu$.

One thus finds, after properly generalizing the results of [6] to the case of an external nonAbelian gauge field, the following expression for the truncated-connected propagator in the eikonal approximation:

$$
\begin{aligned}
& \tilde{G}_{t}\left(p, p^{\prime} \mid A\right) \simeq 2 E \int d^{3} b e^{i q b} \times \\
& \quad\left(P \exp \left[-i g \int_{-\infty}^{+\infty} A_{\mu}(b+p \tau) p^{\mu} d \tau\right]-\mathbf{1}\right)(4)
\end{aligned}
$$

As said before, $q=p^{\prime}-p$ is the transferred momentum. In " $d^{3} b$ " one must not include the component of $b^{\mu}$ which is parallel to $p^{\mu}$. For example, if $p^{\mu} \simeq p^{\prime \mu} \simeq(E, E, 0,0)$, one has that $p_{+} \simeq p_{+}^{\prime} \simeq 2 E$ and $p_{-} \simeq p_{-}^{\prime} \simeq 0$, where the general notation $A_{+} \equiv A^{0}+A^{1}, A_{-} \equiv A^{0}-A^{1}$ has been used for a given four-vector $A^{\mu}$. In this case one must take: $d^{3} b=d^{2} \mathbf{b}_{t} d b_{-}$, where $\mathbf{b}_{t}=\left(b_{y}, b_{z}\right)$ is the component of $b^{\mu}$ in the transverse plane $(y, z)$ (while the two light-cone coor- dinates, $x_{+}=t+x$ and $x_{-}=t-x$, are sometimes called longitudinal coordinates).

Knowing the truncated-connected propagators, one can then extract, using the reduction equations of LSZ in the framework of a functional integral approach, the scattering amplitude of two high-energy spin- 0 quarks, in the limit $s \rightarrow \infty$ and $t \ll s$. The results can then be easily extended to the case of physical interest, i.e., the case of "real" (fermion) QCD.

The quark-quark scattering amplitude, at high squared energies $s$ in the center of mass and small squared transferred momentum $t$ (that is $s \rightarrow \infty$ and $|t| \ll s$, let us say $\left.|t| \leq 1 \mathrm{GeV}^{2}\right)$, turns out to be described by the expectation value of two lightlike Wilson lines, running along the classical trajectories of the two colliding particles [3] [4].

In the center-of-mass reference system (c.m.s.), taking for example the initial trajectories of the two quarks along the $x^{1}$-axis, the scattering amplitude has the following form [explicitly indicating the color indices $(i, j, \ldots)$ and the spin indices $(\alpha, \beta, \ldots)$ of the quarks]

$$
\begin{aligned}
M_{f i} & =\left\langle\psi_{i \alpha}\left(p_{1}^{\prime}\right) \psi_{k \gamma}\left(p_{2}^{\prime}\right)|M| \psi_{j \beta}\left(p_{1}\right) \psi_{l \delta}\left(p_{2}\right)\right\rangle \\
& \sim \underset{s \rightarrow \infty}{\sim}-\frac{i}{Z_{\psi}^{2}} \cdot \delta_{\alpha \beta} \delta_{\gamma \delta} \cdot 2 s \int d^{2} \mathbf{z}_{t} e^{i \mathbf{q} \cdot \mathbf{z}_{t}} \times \\
& \times\left\langle\left[W_{1}\left(z_{t}\right)-\mathbf{1}\right]_{i j}\left[W_{2}(0)-\mathbf{1}\right]_{k l}\right\rangle,
\end{aligned}
$$

where $q=(0,0, \mathbf{q})$, with $t=q^{2}=-\mathbf{q}^{2}$, is the tranferred four-momentum and $z_{t}=\left(0,0, \mathbf{z}_{t}\right)$, with $\mathbf{z}_{t}=\left(z^{2}, z^{3}\right)$, is the distance between the two trajectories in the transverse plane. The expectation value $\langle f(A)\rangle$ is the average of $f(A)$ in the sense of the functional integration over the gluon field $A^{\mu}$ (including also the determinant of the fermion matrix, i.e., $\operatorname{det}\left[i \gamma^{\mu} D_{\mu}-m\right]$, where $D^{\mu}=\partial^{\mu}+i g A^{\mu}$ is the covariant derivative) [3] [4]. The two lightlike Wilson lines $W_{1}\left(z_{t}\right)$ and $W_{2}(0)$ in Eq. (5) are defined as

$$
\begin{aligned}
& W_{1}\left(z_{t}\right)=P \exp \left[-i g \int_{-\infty}^{+\infty} A_{\mu}\left(z_{t}+p_{1} \tau\right) p_{1}^{\mu} d \tau\right] \\
& W_{2}(0)=P \exp \left[-i g \int_{-\infty}^{+\infty} A_{\mu}\left(p_{2} \tau\right) p_{2}^{\mu} d \tau\right]
\end{aligned}
$$

where $P$ stands for "path ordering" and $A_{\mu}=$ $A_{\mu}^{a} T^{a} ; p_{1} \simeq\left(E, E, \mathbf{0}_{t}\right)$ and $p_{2} \simeq\left(E,-E, \mathbf{0}_{t}\right)$ 


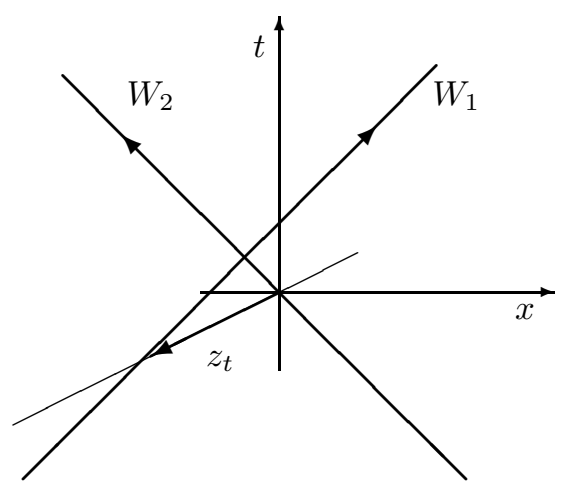

Fig. 1. The space-time configuration of the two lightlike Wilson lines $W_{1}$ and $W_{2}$ entering in the expression (5) for the high-energy quark-quark elastic scattering amplitude.

are the initial four-momenta of the two quarks. The space-time configuration of these two Wilson lines is shown in Fig. 1.

Finally, $Z_{\psi}$ in Eq. (5) is the fermion-field renormalization constant, which can be written in the eikonal approximation as [3]

$Z_{\psi} \simeq \frac{1}{N_{c}}\left\langle\operatorname{Tr}\left[W_{1}(0)\right]\right\rangle=\frac{1}{N_{c}}\left\langle\operatorname{Tr}\left[W_{2}(0)\right]\right\rangle$.

In a perfectly analogous way one can also derive the high-energy scattering amplitude in the case of the Abelian group $U(1)$ (QED). The resulting amplitude is equal to Eq. (5), with the only obvious difference being that now the Wilson lines $W_{1}$ and $W_{2}$ are functionals of the Abelian field $A^{\mu}$ (so they are not matrices). Thanks to the simple form of the Abelian theory (in particular to the absence of self-interactions among the vector fields), it turns out that it is possible to explicitly evaluate (at least in the quenched approximation) the expectation value of the two Wilson lines [4]: one finally recovers the well-known result for the eikonal amplitude of the high-energy scattering in QED [8] [9] [10].

In what follows, we shall deal with the quantity

$g_{M(i j, k l)}(s ; t) \equiv \frac{1}{Z_{\psi}^{2}} \int d^{2} \mathbf{z}_{t} e^{i \mathbf{q} \cdot \mathbf{z}_{t}} \times$

$$
\times\left\langle\left[W_{1}\left(z_{t}\right)-\mathbf{1}\right]_{i j}\left[W_{2}(0)-\mathbf{1}\right]_{k l}\right\rangle,
$$

in terms of which the scattering amplitude can be written as

$$
\begin{aligned}
M_{f i} & =\left\langle\psi_{i \alpha}\left(p_{1}^{\prime}\right) \psi_{k \gamma}\left(p_{2}^{\prime}\right)|M| \psi_{j \beta}\left(p_{1}\right) \psi_{l \delta}\left(p_{2}\right)\right\rangle \\
& \underset{s \rightarrow \infty}{\sim}-i \cdot 2 s \cdot \delta_{\alpha \beta} \delta_{\gamma \delta} \cdot g_{M(i j, k l)}(s ; t) .
\end{aligned}
$$

The quantity $g_{M(i j, k l)}(s ; t)$ depends not only on $t=-\mathbf{q}^{2}$, but also on $s$. In fact, as was pointed out by Verlinde and Verlinde in 11, it is a singular limit to take the Wilson lines in (8) exactly lightlike. A way to regularize this sort of "infrared" divergence (so called because it essentially comes from the limit $m \rightarrow 0$, where $m$ is the quark mass) consists in letting each line have a small timelike component, so that they coincide with the classical trajectories for quarks with a finite mass $m$ (see also Ref. [12] for a discussion about this point). In other words, one first evaluates the quantity $g_{M(i j, k l)}(\beta ; t)$ for two Wilson lines along the trajectories of two quarks (with mass $m$ ) moving with velocity $\beta$ and $-\beta(0<\beta<1)$ in the $x^{1}$-direction. This is equivalent to consider two infinite Wilson lines forming a certain (finite) hyperbolic angle $\chi$ in Minkowski space-time. Then, to obtain the correct high-energy scattering amplitude, one has to perform the limit $\beta \rightarrow 1$, that is $\chi \rightarrow \infty$, in the expression for $g_{M(i j, k l)}(\beta ; t)$ :

$$
\begin{aligned}
& M_{f i}=\left\langle\psi_{i \alpha}\left(p_{1}^{\prime}\right) \psi_{k \gamma}\left(p_{2}^{\prime}\right)|M| \psi_{j \beta}\left(p_{1}\right) \psi_{l \delta}\left(p_{2}\right)\right\rangle \\
& \underset{s \rightarrow \infty}{\sim}-i \cdot 2 s \cdot \delta_{\alpha \beta} \delta_{\gamma \delta} \cdot g_{M(i j, k l)}(\beta \rightarrow 1 ; t)(10)
\end{aligned}
$$

Proceeding in this way one obtains a $\ln s$ dependence of the amplitude, as expected from ordinary perturbation theory and as confirmed by the experiments on hadron-hadron scattering processes [1] [2].

For deriving the dependence on $s$ one exploits the fact that both $\beta$ and $\chi$ are dependent on $s$. In fact, from $E=m / \sqrt{1-\beta^{2}}$ and from $s=4 E^{2}$, one immediately finds that

$\beta=\sqrt{1-\frac{4 m^{2}}{s}}$.

By inverting this equation and using the relation $\beta=\tanh \psi$, where $\psi$ is the hyperbolic angle [in the plane $\left.\left(x^{0}, x^{1}\right)\right]$ of the trajectory of $W_{1}$, we derive that

$s=4 m^{2} \cosh ^{2} \psi=2 m^{2}(\cosh \chi+1)$. 
Therefore, in the high-energy limit $s \rightarrow \infty$ (or $\beta \rightarrow 1$ ), the hyperbolic angle $\chi=2 \psi$ is essentially equal to the logarithm of $s$ (for a finite non-zero quark mass $m$ ):

$\chi=2 \psi \underset{s \rightarrow \infty}{\sim} \ln s$.

In Sect. 3 of Ref. [12] we have followed this procedure to explicitly evaluate the second member of (10) up to the fourth order in the expansion in the renormalized coupling constant $g_{R}$ : the results so derived are in agreement with those obtained by applying ordinary perturbation theory to evaluate the scattering amplitude up to the order $O\left(g_{R}^{4}\right)$ [1] [2].

The direct evaluation of the expectation value (8) is a highly non-trivial matter and it is strictly connected with the ultraviolet properties of Wilson-line operators [13]. Some nonperturbative approaches for the calculation of (8) have been proposed in Refs. [14] and [15]. At the moment, the only non-perturbative numerical estimate of (8), which can be found in the literature, is that of Ref. 115] (where it has been generalized to the case of hadron-hadron scattering): it has been obtained in the framework of the stochastic vacuum model (SVM).

In some recent papers [12] [16] we have proposed a new approach, which consists in adapting the scattering amplitude to the Euclidean world: this approach could open the way for the direct evaluation of the scattering amplitude on the lattice. More explicitly, we have shown that the expectation value of two infinite Wilson lines, forming a certain hyperbolic angle in Minkowski space-time, and the expectation value of two infinite Euclidean Wilson lines, forming a certain angle in Euclidean four-space, are connected by an analytic continuation in the angular variables.

Let us consider the following quantity, defined in Minkowski space-time:

$$
\begin{aligned}
& g_{M}\left(p_{1}, p_{2} ; t\right)=\frac{1}{Z_{W}^{2}} \int d^{2} \mathbf{z}_{t} e^{i \mathbf{q} \cdot \mathbf{z}_{t}} \times \\
& \quad \times\left\langle\left[W_{1}\left(z_{t}\right)-\mathbf{1}\right]_{i j}\left[W_{2}(0)-\mathbf{1}\right]_{k l}\right\rangle,
\end{aligned}
$$

where $p_{1}$ and $p_{2}$ are the four-momenta [lying (for example) in the plane $\left.\left(x^{0}, x^{1}\right)\right]$, which define the trajectories of the two Wilson lines $W_{1}$ and $W_{2}$
( $A_{\mu}=A_{\mu}^{a} T^{a}$ and $m$ is the fermion mass):

$$
\begin{aligned}
& W_{1}\left(z_{t}\right) \equiv P \exp \left[-i g \int_{-\infty}^{+\infty} A_{\mu}\left(z_{t}+\frac{p_{1}}{m} \tau\right) \frac{p_{1}^{\mu}}{m} d \tau\right], \\
& W_{2}(0) \equiv P \exp \left[-i g \int_{-\infty}^{+\infty} A_{\mu}\left(\frac{p_{2}}{m} \tau\right) \frac{p_{2}^{\mu}}{m} d \tau\right] .(15)
\end{aligned}
$$

$Z_{W}$ in Eq. (14) is defined as

$Z_{W} \equiv \frac{1}{N_{c}}\left\langle\operatorname{Tr}\left[W_{1}(0)\right]\right\rangle=\frac{1}{N_{c}}\left\langle\operatorname{Tr}\left[W_{2}(0)\right]\right\rangle$.

This is a sort of Wilson-line's renormalization constant: as shown in Ref. [3], $Z_{W}$ coincides with the fermion renormalization constant $Z_{\psi}$ in the eikonal approximation.

By virtue of the Lorentz symmetry, we can define $p_{1}$ and $p_{2}$ in the c.m.s. of the two particles, moving with speed $\beta$ and $-\beta$ along the $x^{1-}$ direction: the hyperbolic angle $\chi$ [in the plane $\left.\left(x^{0}, x^{1}\right)\right]$ between the two trajectories of $W_{1}$ and $W_{2}$ is thus given by $\chi=2 \operatorname{arctanh} \beta$.

In an analogous way, we can consider the following quantity, defined in Euclidean space-time:

$$
\begin{aligned}
& g_{E}\left(p_{1 E}, p_{2 E} ; t\right)=\frac{1}{Z_{W E}^{2}} \int d^{2} \mathbf{z}_{t} e^{i \mathbf{q} \cdot \mathbf{z}_{t}} \times \\
& \quad \times\left\langle\left[W_{1 E}\left(z_{t E}\right)-\mathbf{1}\right]_{i j}\left[W_{2 E}(0)-\mathbf{1}\right]_{k l}\right\rangle_{E},
\end{aligned}
$$

where $z_{t E}=\left(z_{1}, z_{2}, z_{3}, z_{4}\right)=\left(0, \mathbf{z}_{t}, 0\right)$ and $q_{E}=$ $(0, \mathbf{q}, 0)$ (so that: $\left.q_{E}^{2}=\mathbf{q}^{2}=-t\right)$. The expectation value $\langle\ldots\rangle_{E}$ must be intended now as a functional integration with respect to the gauge variable $A_{\mu}^{(E)}=A_{\mu}^{(E) a} T^{a}$ in the Euclidean theory. The Euclidean four-vectors $p_{1 E}$ and $p_{2 E}$ [lying (for example) in the plane $\left.\left(x_{1}, x_{4}\right)\right]$ define the trajectories of the two Euclidean Wilson lines $W_{1 E}$ and $W_{2 E} . Z_{W E}$ in Eq. (17) is defined analogously to $Z_{W}$ in Eq. (16):

$Z_{W E} \equiv \frac{1}{N_{c}}\left\langle\operatorname{Tr}\left[W_{1 E}(0)\right]\right\rangle=\frac{1}{N_{c}}\left\langle\operatorname{Tr}\left[W_{2 E}(0)\right]\right\rangle$.

Denoting by $\theta$ the Euclidean angle in the plane $\left(x_{1}, x_{4}\right)$ between the trajectories of the two Euclidean Wilson lines $W_{1 E}$ and $W_{2 E}$, one finally finds the following relation between the amplitudes $g_{M}(\chi ; t)$ and $g_{E}(\theta ; t)$ 16]:

$$
\begin{aligned}
& g_{M}(\chi ; t) \underset{\chi \rightarrow i \theta}{\longrightarrow} g_{M}(i \theta ; t)=g_{E}(\theta ; t) ; \\
& g_{E}(\theta ; t) \underset{\theta \rightarrow-i \chi}{\longrightarrow} g_{E}(-i \chi ; t)=g_{M}(\chi ; t) .
\end{aligned}
$$


This relation of analytic continuation has been proven in Ref. 12] for an Abelian gauge theory (QED) in the so-called quenched approximation and for a non-Abelian gauge theory (QCD) up to the fourth order in the renormalized coupling constant in perturbation theory. In Ref. [16], we have generalized the results of Ref. [12] giving the rigorous proof of the above-mentioned relation of analytic continuation for a non-Abelian gauge theory with gauge group $S U\left(N_{c}\right)$ [as well as for an Abelian gauge theory (QED)]. The approach adopted in Ref. 12 consisted in explicitly evaluating the amplitudes $g_{M}$ and $g_{E}$ in some given approximation (such as the quenched approximation) or up to some order in perturbation theory and in finally comparing the two expressions so obtained. Instead, in Ref. [16] we have given a general proof, which essentially exploits the relation between the gluonic Green functions in the two theories.

Therefore, it is possible to reconstruct the high-energy scattering amplitude by evaluating a correlation of two infinite Wilson lines forming a certain angle $\theta$ in Euclidean four-space, then by continuing this quantity in the angular variable, $\theta \rightarrow-i \chi$, where $\chi$ is the hyperbolic angle between the two Wilson lines in Minkowski spacetime, and finally by performing the limit $\chi \rightarrow \infty$ (i.e., $\beta \rightarrow 1$ ). In fact, the high-energy scattering amplitude is given by

$$
\begin{aligned}
& M_{f i}=\left\langle\psi_{i \alpha}\left(p_{1}^{\prime}\right) \psi_{k \gamma}\left(p_{2}^{\prime}\right)|M| \psi_{j \beta}\left(p_{1}\right) \psi_{l \delta}\left(p_{2}\right)\right\rangle \\
& \underset{s \rightarrow \infty}{\sim}-i \cdot 2 s \cdot \delta_{\alpha \beta} \delta_{\gamma \delta} \cdot g_{M}(\chi \rightarrow \infty ; t) .
\end{aligned}
$$

The quantity $g_{M}(\chi ; t)$, defined by Eq. (14) in the Minkowski world, is linked to the corresponding quantity $g_{E}(\theta ; t)$, defined by Eq. (17) in the Euclidean world, by the analytic continuation (19) in the angular variables. The important thing to note here is that the quantity $g_{E}(\theta ; t)$, defined in the Euclidean world, may be computed non perturbatively by well-known and well-established techniques, for example by means of the formulation of the theory on the lattice or using the SVM 15. In all cases, once one has obtained the quantity $g_{E}(\theta ; t)$, one still has to perform an analytic continuation in the angular variable $\theta \rightarrow-i \chi$, and finally one has to extrapolate to the limit $\chi \rightarrow \infty$ (i.e., $\beta \rightarrow 1$ ). For deriving the dependence on $s$ one exploits the fact that both $\beta$ and $\chi$ are dependent on $s$, according to Eqs. (11), (12) and (13).

Of course, the most interesting results are expected from an exact non perturbative approach, for example by directly computing $g_{E}(\theta ; t)$ on the lattice: a considerable progress could be achieved along this direction in the near future.

\section{REFERENCES}

1. H. Cheng and T.T. Wu, Expanding Protons: Scattering at High Energies (MIT Press, Cambridge, Massachussets, 1987).

2. L.N. Lipatov, in Review in Perturbative $Q C D$, edited by A.H. Mueller (World Scientific, Singapore, 1989), and references therein.

3. O. Nachtmann, Ann. Phys. 209, 436 (1991).

4. E. Meggiolaro, Phys. Rev. D 53, 3835 (1996).

5. E.S. Fradkin, Proceedings of the International Winter School on Theoretical Physics at JINR (Dubna, 1964); Acta Phys. Hung. XIX, 175 (1964).

6. M. Fabbrichesi, R. Pettorino, G. Veneziano and G.A. Vilkovisky, Nucl. Phys. B419, 147 (1994).

7. R.P. Feynman, Rev. Mod. Phys. 20, 327 (1948).

8. H. Cheng and T.T. Wu, Phys. Rev. Lett. 22, 666 (1969).

9. H. Abarbanel and C. Itzykson, Phys. Rev. Lett. 23, 53 (1969).

10. R. Jackiw, D. Kabat and M. Ortiz, Phys. Lett. B 277, 148 (1992).

11. H. Verlinde and E. Verlinde, Princeton University, report No. PUPT-1319 (revised 1993); hep-th/9302104.

12. E. Meggiolaro, Pisa preprint, IFUP-TH 10/96 (1996); hep-th/9602104; to appear in Zeitschrift für Physik C.

13. I.Ya. Aref'eva, Phys. Lett. 93B, 347 (1980).

14. I.Ya. Aref'eva, Phys. Lett. B 325, 171 (1994); 328, 411 (1994).

15. H.G. Dosch, E. Ferreira and A. Krämer, Phys. Rev. D 50, 1992 (1994).

16. E. Meggiolaro, Pisa preprint, IFUP-TH $9 / 97$ (1997); hep-th/9702186. 


\section{DISCUSSIONS}

L.N. Lipatov, INP, St. Petersburg (Russia) What is the relation of your work with the BFKL results (LLA for high-energy scattering)? In 1986 the quark-quark scattering amplitudes with the elastic and quasi-elastic unitarity were constructed. Are they related with your eikonal amplitude?

\section{E. Meggiolaro}

It is known that "soft" hadronic collisions at high energy can be described by the Pomeron-exchange model. However, a more basic understanding of Pomeron exchange in terms of the underlying quark-gluon dynamics (QCD) remains an outstanding open problem. Most theorists now agree that the properties of the Pomeron can be somehow derived in QCD from multi-gluon exchange and the physical picture commonly accepted is the one where the Pomeron couples to single partons: yet, it is still unclear how to develop a reliable calculational framework based on this idea. The first calculations of the near-forward parton-parton scattering amplitudes were done to the lowest orders of perturbation theory (PT) in the leading logarithmic approximation (LLA), or using the elastic and quasi-elastic unitarity. In particular, the high-energy asymptotics of the scattering amplitude was found in the LLA, using the results of one-loop calculations in order to resum the leading logarithmic corrections to all oders of PT. However, in spite of the considerable progress so achieved, there was no regular way to resum and control non-leading logarithmic corrections to the scattering amplitude.

The approach described in my work enables us to take into account systematically these nonleading corrections. It starts from the first principles of $Q C D$ and derives the high-energy quark-quark scattering amplitude in a fully nonperturbative way, using a functional integral approach and the so-called eikonal approximation to write the truncated-connected quark propagator in an external gluon field. A comparison of this approach to the standard PT results has been performed up to the one-loop level (fourth order in the renormalized coupling con- stant): the two approaches give the same results. An explicit comparison with the BFKL Pomeron model is not yet available. It would be an extremely important result to derive the features of the Pomeron model starting from my nonperturbative approach, based on first principles of $Q C D$ : this is matter for future work. 